\title{
Discussion on Optimization of Public Transportation Network Setting considering Three-State Reliability
}

\author{
Zhang Xiaoliang $\mathbb{D}^{1,2}$ and Jia Limin $\mathbb{D}^{1}$ \\ ${ }^{1}$ School of Traffic and Transportation, Beijing Jiaotong University, Beijing 100044, China \\ ${ }^{2}$ Research Institute of Highway, Ministry of Transport, No. 8 Xi Tucheng Road, Haidian District, Beijing 100088, China
}

Correspondence should be addressed to Zhang Xiaoliang; 16114226@bjtu.edu.cn

Received 5 June 2021; Accepted 10 July 2021; Published 26 July 2021

Academic Editor: Qi-zhou Hu

Copyright (c) 2021 Zhang Xiaoliang and Jia Limin. This is an open access article distributed under the Creative Commons Attribution License, which permits unrestricted use, distribution, and reproduction in any medium, provided the original work is properly cited.

\begin{abstract}
In order to be environment-friendly, relieve traffic congestion, reduce pollution, and be green and sustainable, the optimization and development of public transportation, as the subject of people's long-term research, has always been shining. With the emergence of shared transportation, public transportation systems face more challenges. In order to better connect with bikesharing, car-sharing, and other modes of transportation, public transportation will carry out important reforms, among which the optimization of line network is one of the most important tasks. The traditional bus route design is mainly based on the "fourstage" method model, which is mainly based on the investigation and analysis of the existing traffic system and land use. Through the work flow of "evaluation, calibration, and verification," the network balance optimization model is used to get the bus travel allocation prediction model. In this paper, the optimization problem of public transit network is studied from the point of view of the reliability of public transit network. It is proposed that public transit network can be abstracted into series-parallel system and parallel-series system model from the three states of normal, short-circuit failure, and open-circuit failure and is analyzed and discussed through the hypothesis experiment. The research of this paper will provide a new perspective for the optimization of public transit network, complement the traditional methods, and support the optimization and reliability improvement of urban public transit network. More reliable bus networks and other modes of transportation, such as walking, bike-sharing, and rail, will become more suitable for people to get around.
\end{abstract}

\section{Introduction}

For better sustainable development of cities and urban traffic, in 2011, China launched a national "bus city" project [1]. There were more cities that took part in this project such as Beijing, Shanghai, Hangzhou, and Suzhou. With the emergence of shared transportation, public transportation systems face more challenges. In order to better connect with bike-sharing, car-sharing, and other modes of transportation, public transportation will carry out important reforms, among which the optimization of line network is one of the most important tasks. At the same time, the action of remolding the ground bus network successively appeared in many major cities around the world, such as Seoul, Barcelona, Sydney, Berlin, Houston, Baltimore, Dallas,
Portland, Los Angeles, New York, Paris, and London [2-7]. As people's travel demand becomes higher and higher, bus network reliability plays a very important role in people's bus service experience [8] due to the influence of various factors on bus operation. Therefore, it is necessary to combine the reliability of bus network with the optimized setting of bus network for analysis and research. It provides a comprehensive decision-making method for improving the network topology structure and actual operation of public transit network [9].

Research on the structure of public bus network and the optimization of network stations mainly focuses on the network organization forms of bus lines, such as radial and square grid $[10,11]$. As early as 1989, Wang Wei put forward a simple and practical method of "laying out bus 
lines one by one and optimizing them into a network," which was based on the optimization of single bus lines [12]. Later, some scholars proposed the classification and layering of bus routes according to different passenger flow transportation to be completed, including the radiation-type network system [13], the design of bus corridors, and the radiation-shaped long-distance transportation network. Some scholars proposed the structure of mixed bus network, such as the main network, secondary network, and transfer network [14, 15]. Based on the concept of hierarchical network, multimode network design method [16], fuzzy logic design method, and systematic method are proposed [17]. Another scholar proposed sensitivity analysis of the bus network and established three types of bus networks: transfer guidance network, transfer avoidance network, and direct connection network $[18,19]$.

Conventional bus route optimization methods mainly include mathematical analysis model and optimization algorithm [20]. Mathematical analysis is used to study the route configuration process, including traffic allocation. OD of residents' buses is reasonably allocated to the bus network to form the optimal flow distribution. Under certain constraints, various optimization methods are used to generate the optimal route network $[21,22]$. The traditional optimization method is to establish the optimization model. The optimization model usually takes the following indicators as the optimization objectives, including the minimum transfer coefficient, the optimal nonlinear coefficient of the line, the highest line-network coverage, the minimum travel cost of bus passengers, and operation cost [23]. With the development of search algorithm and computer technology, heuristic algorithm, genetic algorithm, combined simulated annealing, tabu search algorithm, and so on have all become popular algorithms to solve the problem of line network optimization [24-28].

Many scholars have studied the reliability of bus networks, among which the most commonly used ones are those based on the reliability of road network, mainly including the connectivity of road network, the reliability of travel time, the reliability of road network capacity, and the reliability of unblocked traffic [29-33]. Some scholars have used graph theory to study the reliability of bus network connectivity $[34,35]$, as well as studies on the reliability of running time [36]. However, there are not many achievements on the combination of bus reliability and optimization design of line network [37].

To sum up, most public transit network optimization methods are based on the hierarchical view of existing bus operation functions and the path optimization based on the existing road network. For the topology of the public transport network, a relatively macroscopic study is carried out from the shape composition of the network. In the aspect of bus network reliability, researches are mainly based on road network reliability, network connectivity, reliability of running time, evaluation indexes of bus operation reliability, and so forth, but there are few researches on bus network reliability and more detailed bus network composition [38-40].
In this paper, the composition and setting of bus corridor system in the topology of bus network are considered from the meso point of view. In the study of the problem of considering the design of bus line network constituted by bus corridors, the calculation method of reliability of line network is introduced [34]. In the process of constructing the topology structure model of the bus corridor, which is the meso component of the bus network, the complex bus network is abstracted into the basic units composed of bus lines, stations, and interstation paths, which are series system and parallel system [41]. Then the reliability of the different basic unit combination study includes series-parallel system and parallel-series system research. "Normal operation," "open-circuit failure," and "short-circuit failure" theory-oriented and structural reliabilities under three states relations are discussed; and we combine with the actual public transport corridor in the channel as the research scene, by using the number of series subsystems and the number of parallel subsystems in the subsystem. The simulation experiment is carried out, and the optimization conclusion of general bus corridor network setting based on tri-state reliability is obtained.

The remainder of the paper is organized as follows: Section 2 is the construction of the reliability model of public transit network, that is, bus corridor network; Section 3 is the simulation analysis of series-parallel system; Section 4 is the simulation analysis of parallel-series system; Section 5 is the conclusion and discussion. Through the exploration and research on the more basic topological level, this paper provides an innovative idea for the optimization of bus network setting, which is a supplement to the traditional method. Combined with the actual operation of the lines, it will play an important practical significance for the optimization and adjustment of the subsequent bus network and the design of bus corridors.

\section{Bus Line System Reliability Modeling}

Step 1: bus line network can be regarded as a network composed of bus lines at different starting points, which can be expressed by a simple schematic diagram as Figure 1.

Step 2: separate the bus corridors at arbitrary starting and finishing points, which can be seen as a network diagram composed of bus stations and interstation paths and can be expressed in a simple schematic diagram as Figure 2.

Step 3 (abstract classification of the network): bus line network can be composed of series and parallel bus lines; the bus corridor can be composed of series and parallel paths between bus stations, in which a single bus line is composed of series paths between bus stations.

Step 4: the network characteristics of public transit network are composed of series line characteristics and parallel line characteristics. Assuming that each interstation path is a subsystem, it will be decomposed into the two following basic types: 


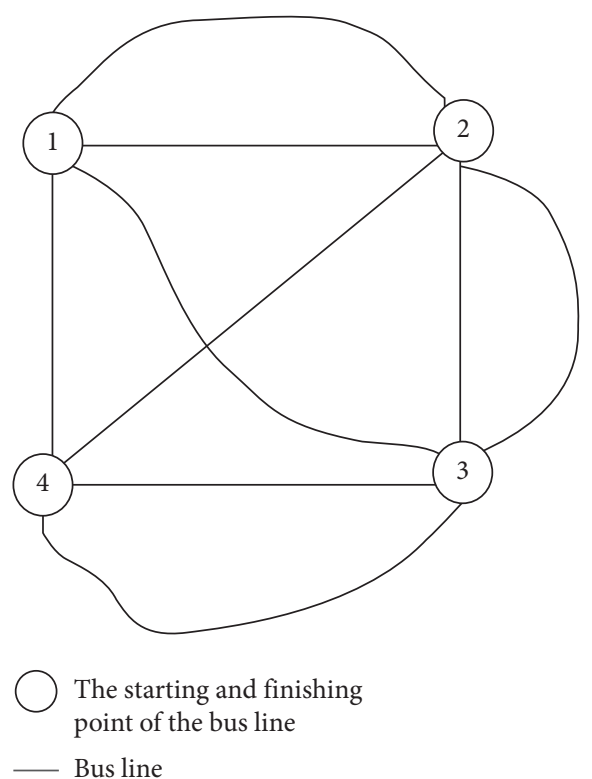

FIGURE 1: Schematic diagram of the line network constituted by bus routes.

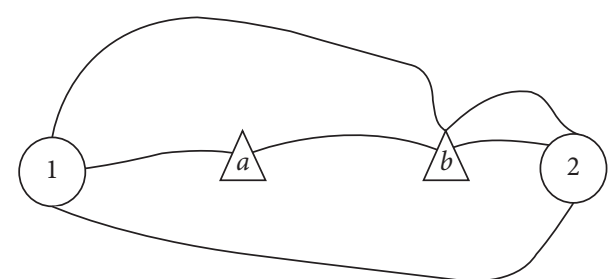

The starting and finishing point of the bus line

$$
\begin{aligned}
& \triangle \text { A stop in a bus line } \\
& \text { The interstation path between the } \\
& \text { stops of a bus route }
\end{aligned}
$$

Figure 2: Schematic diagram of bus corridor network formed by routes between bus stations.

(1) Series-parallel system: it is assumed that the system is composed of a series of $n$ identical three-state subsystems (i.e., redundant set of interstation subsystems), and each subsystem is composed of parallel $m$ different three-state interstation path subsystems as in Figure 3

(2) Parallel-series system: it is assumed that the system is composed of $m$ identical three-state subsystems (i.e., bus lines) in parallel, and each subsystem is composed of $n$ different three-state station path subsystems in series as in Figure 4

Step 5: consider the reliability problem of public transit network; that is, it is transformed into the reliability problem of series-parallel system and parallel-series system.

Hypothesis: there are three states of the basic unit of bus network-interstation path: normal state, open-circuit failure state, and short-circuit failure state.

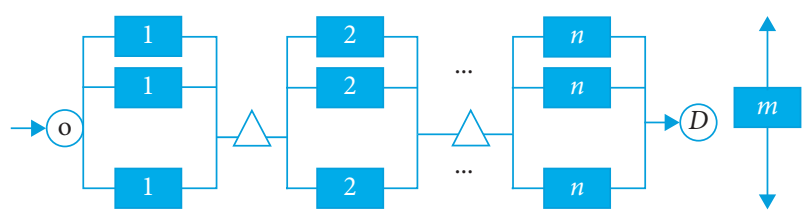

Figure 3: Schematic diagram of the series-parallel system.

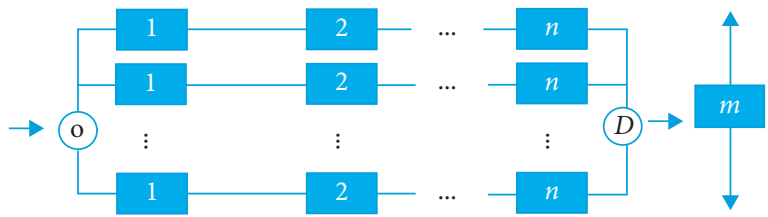

FIGURE 4: Schematic diagram of the parallel-series system.

Consider the following:

(1) The normal state refers to the completion of the passenger flow transportation task on the specified line at the specified time.

(2) The open-circuit failure state is that the bus operation fails to complete the passenger transport task within a certain time range and greatly exceeds the interstation running time, making both ends of the paths between bus stations like electronic components open circuit. In practice, "large interval" is a common situation in the interstation path system. Open road conditions that can lead to travel are more severe in the event of an accident or in the event of bad weather or road disruption.

(3) Short-circuit failure status is that the bus runs in a certain path range and does not complete the established passenger transport task. The bus operates as if the electric current is short-circuiting without passing through the load that the passengers are like loads. As the next bus arrived too close to the station, almost no passengers got on. In the actual public transport system, "bunching" is such a common phenomenon. If no new passengers are transported at the passing station, this section will be invalid.

\section{Reliability Analysis and Simulation Experiment of the Series-Parallel System}

3.1. Reliability Analysis of the Series-Parallel System. The series-parallel system is very common in the actual bus corridor. There are many alternative bus routes between stations in the corridor. The set of alternative routes between stations constitutes each parallel subsystem, and the whole bus corridor is series-parallel system.

For the convenience of calculation and analysis, it is assumed that the operating conditions of each interstation path are the same, and the open-circuit failure probability of each interstation path is set as $q_{o}$ and the short-circuit failure probability is set as $q_{s}$; then each interstation path is parallel subsystem.

The open-circuit failure probability is 


$$
Q_{0}=\prod_{i=1}^{m} q_{0 i}
$$

The short-circuit failure probability is

$$
Q_{s}=1-\prod_{i=1}^{m}\left(1-q_{s i}\right)
$$

The reliability of bus corridor series-parallel system is

$$
R_{s}=\left(1-Q_{0}\right)^{n}-Q_{s}^{n}=\left(1-\prod_{i=1}^{m} q_{0 i}\right)^{n}-\left[1-\prod_{i=1}^{m}\left(1-q_{s i}\right)\right]^{n}
$$

3.2. Simulation Experiment of the Series-Parallel System. It is assumed that the operation conditions of the paths between stations are the same and that the open-circuit failure probability of the paths between stations is $q_{o}=0.15$ and the short-circuit failure probability is $q_{s}=0.05$ in the three states according to the experience. The simulation experiment of series-parallel system is carried out:

(1) Fix the value of $n$, set the parameter $n=[2,3,4,5]$, observe the trend of system reliability changing with $m$, realize by programming language, and generate the following figure:

In Figure 5, which is the reliability of series-parallel system, when $n$ is fixed, the reliability $R_{s}$ varies with $m$. As shown in the figure, the curves basically reach the maximum near $m=5$, and then $R_{s}$ decreases with the increase of $m$.

(2) Fix the value of $m$, set the parameter $m=[2,3,4,5]$ which is used to observe the trend of system reliability changing with $n$ and is realized by programming language to generate Figure 6.

In Figure 6, the reliability of series-parallel system is fixed $m$, where $m=[2,3,4,5]$. With the increase of $n, R_{s}$ first increases and reaches the maximum value at $n=5$. After that, with the increase of $n, R_{s}$ has little change. According to Figures 5 and 6 , the reliability of the three-state seriesparallel system composed of the same interstation path reaches the maximum when both $m$ and $n$ are equal to 5 .

\section{Reliability Analysis and Simulation Experiment of the Parallel-Series System}

4.1. Reliability Analysis of the Parallel-Series System. Parallel-series system in actual transit corridor or theoryoriented and large passenger transport channel is very common. There are a lot of alternative bus routes selection between the starting station and the finishing station. Each bus line is made of the path between the station subsystem and series subsystem. The alternative set of bus lines make up a large parallel redundant system. The network of the entire bus corridor or large passenger passageway bus lines is a parallel-series system.

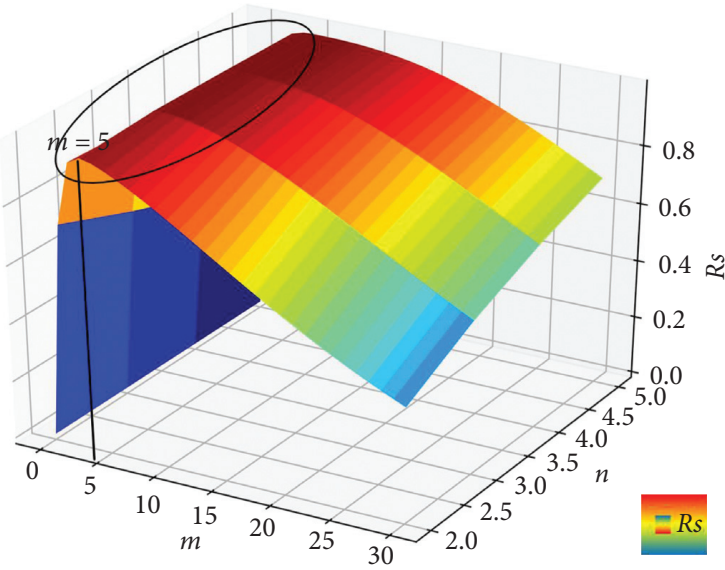

FIgURE 5: Curve of reliability of series and parallel systems changing with parameter $m$.

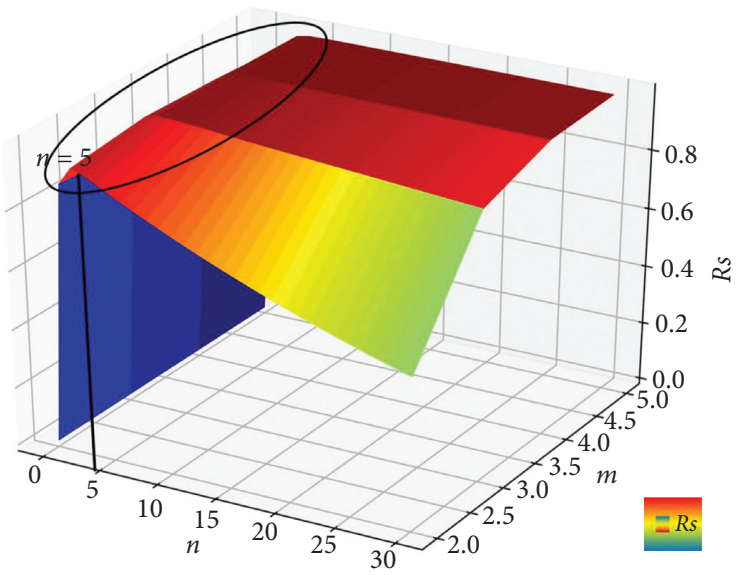

FIGURE 6: Reliability curve of series-parallel system as a function of parameter $n$.

For the convenience of calculation and analysis, it is assumed that the running conditions of each interstation path are the same, and the open-circuit failure probability of each interstation path is set as $q_{o}$, and the short-circuit failure probability is set as $q_{s}$; then each bus line is connected in series subsystem.

The open-circuit failure probability is

$$
Q_{0}=1-\prod_{i=1}^{n}\left(1-q_{0 i}\right)
$$

The short-circuit failure probability is

$$
Q_{s}=\prod_{i=1}^{n} q_{s i}
$$

The bus corridor (large passenger passage) is a parallelseries system, and the system reliability is expressed as

$$
R_{s}=\left(1-Q_{s}\right)^{m}-Q_{0}^{m}=\left(1-\prod_{i=1}^{n} q_{s i}\right)^{m}-\left[1-\prod_{i=1}^{n}\left(1-q_{0 i}\right)\right]^{m} \text {. }
$$


4.2. Simulation Experiment of the Parallel-Series System. In the actual situation, in the layout and optimization process of bus routes in the bus corridor, how many stations, how many interstation routes, and how many bus lines should be laid by using the parallel-series system to make the bus corridor have the highest reliability as follows.

It is assumed that the operation conditions of the paths between stations are the same, and the three-state failure rate values of the paths between stations are assumed according to experience, in which the open-circuit failure probability is $q_{o}=0.15$ and the short-circuit failure probability is $q_{s}=0.05$. The simulation experiment of the parallel-series system is carried out:

(1) Set the parameter $n=[2,3,4,5]$ to observe the trend of system reliability changing with $m$, and realize it through programming language to generate the following figure:

According to Figure 7, the reliability of the parallelseries system is fixed at $n$, and the reliability $R_{s}$ changes with $m$ as shown in the figure. Most of the curves reach the maximum value at $m=5$, and then the reliability basically remains unchanged.

(2) Set the parameter $m=[2,3,4,5]$, observe the trend of system reliability changing with $n$, realize by programming language, and generate Figure 8.

Figures 7 and 8 show the curve of the reliability of the parallel-series system changing with parameter. When $m$ is fixed, $R_{s}$ first increases with the increase of $n$ and reaches the maximum when $n=2$. According to Figures 7 and 8 , the reliability of the parallel-series three-state bus corridor system composed of the same interstation paths reaches its maximum value when $m$ and $n$ are 5 and 2, respectively.

\section{Conclusion and Discussion}

Thus, it can be seen that, without considering other influencing factors, the design of bus corridor basic units with at least five alternative routes is the most reliable. In practice, the route setting and station planning between stations will be determined according to the combination form of the route and station. If it is through each station, then use the serial-parallel system form, and each basic unit 6 stations is appropriate. If the large fast passenger flow channel is used, the use of parallel-series system form can set up three stations in the middle of the line. In the network design of the bus corridor and the large passenger flow channel of the parallel-series system, it is assumed that the operation conditions of the interstation path system are the same, and the reliability of the bus corridor and the large passenger flow channel of the parallel-series system reaches the maximum when $m$ is 5 and $n$ is 2 . The above research shows that, in the design of the bus corridor network system, the reliability of the system is not positively correlated with the number of lines. In the design of the bus corridor of the series-parallel system, the reliability of the bus corridor of the series-parallel system is maximized when $m$ and $n$ are 5,

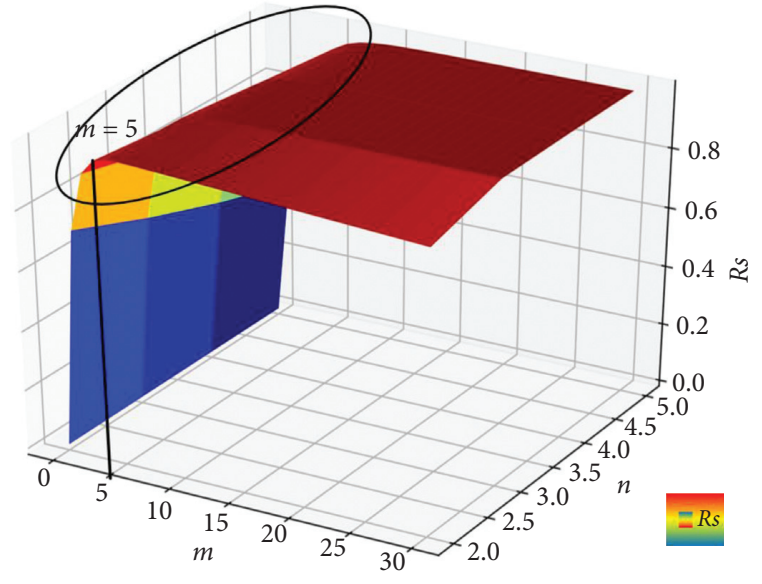

FIGURE 7: Reliability curve of parallel-series system as a function of parameter $m$.

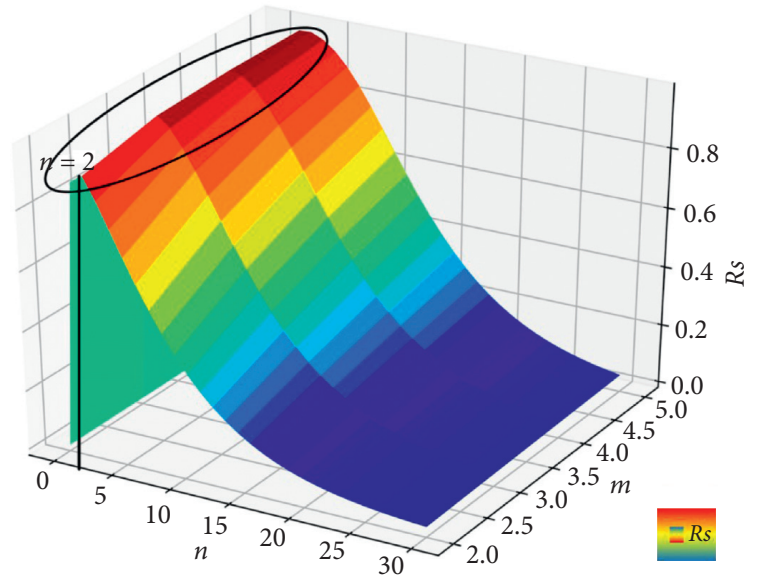

FIGURE 8: Curve of the reliability of tandem system changing with parameter $n$.

assuming that the running conditions of the interstation path system are the same.

In the case of a bus route system that is composed of the same route between stations, it may be a little different from what we normally think of as the more connections, the more reliable the system. In fact, the above experiment shows that the deployment of such a system, for a parallel system $R_{s}=\left(1-q_{s}\right)^{n}-q_{o}^{n}$, takes the partial derivative and sets it equal to zero as follows:

$$
n=\frac{\ln \left[\ln \left(1-q_{s}\right) / \ln q_{0}\right]}{\ln \left(q_{0} /\left(1-q_{s}\right)\right)}
$$

The optimal number of parallel connections $n$ can be determined.

When $q_{o}<q_{s}$ and $n<1$, it is shown that when the shortcircuit failure probability of the interstation path is greater than the open-circuit failure probability, the parallel structure cannot be adopted.

When $q_{o}=q_{s}$ and $n \approx 1$, it is also not suitable for parallel connection. 
When $q_{o}>q_{s}$ and $n>1$, in other words, the reliability of the bus corridor system can be improved by adopting the appropriate number of interstation path redundancies, but the reliability does not monotonically increase with the increase of the number of interstation path redundancies, that is, the number of alternative routes. In other words, there is the problem of optimal redundancy, and the optimal number of redundancies is $n$.

Similarly, the problem of optimal quantity exists in series systems. In order to obtain the optimal number of paths $n$ between stations in series, the method is similar to the processing method of parallel system $R_{s}=\left(1-q_{o}\right)^{n}-q_{s}^{n}$; take the partial derivative and set it equal to zero as follows:

$$
n=\frac{\ln \left[\ln \left(1-q_{0}\right) / \ln q_{s}\right]}{\ln \left(q_{s} /\left(1-q_{o}\right)\right)} .
$$

When $q_{o}=q_{s}$ and $n \approx 1$, the optimal number of series is 1 , and when $q_{o}>q_{s}$ and $n<1$, the optimal number of series is less than 1. It shows that the system should not adopt series structure at this time. It is obvious that the open-circuit failure of interstation paths is greater than short-circuit failure, and the more interstation paths in series, the less reliable the bus route system.

When $q_{o}<q_{s}$ and $n>1$, the optimal series number is greater than 1, the appropriate series structure can improve the system reliability, and the optimal number is $n$.

In general, this method based on online reliability is of good reference significance for the setting and optimization design of bus network from the perspective of bus network topology structure, and the basic laws that constitute the bottom layer of bus network topology structure are found. However, the research in this paper only considers the cases of consistent route operation between stations. In the future research, the study should be carried out according to a variety of conditions of the interstation route. At the same time, the improvement should be made based on the actual road network foundation conditions, road operation conditions, transportation capacity of the actual line operation enterprises, and actual passenger flow demand, which will have better effect and more practical significance. More reliable bus networks and other modes of transportation, such as walking, bike-sharing, and rail, will become more suitable for people to get around.

\section{Data Availability}

The simulation data used to support the study are included within the paper.

\section{Conflicts of Interest}

The authors declare that they have no conflicts of interest.

\section{Acknowledgments}

This research has been funded by the National Key R\&D Plan (project no. 2017YFC0840206) and Technology Project of the Beijing Traffic Commission under Grant 201905ZHJC2.

\section{References}

[1] Ministry of Transport, Notice on the Issues Related to the Demonstration Project of Transit Metropolis, Ministry of Transport, Beijing, China, 2011.

[2] SEG, Seoul Public Transportation, Seoul Metropolitan Government, Seoul, South Korea, 2014.

[3] AdB, Nova Xarxa de Bus de Barcelona, Ajuntament de Barcelona, Barcelona, Spain, 2012.

[4] NSW, Sydney's Bus Future: Simpler, Faster, Better Bus Services, Transport for New South Wales, Sydney, Australian, 2013.

[5] NTA, Dublin Area Bus Network Redesign Public Consultation Report, National Transport Authority, Dublin, Ireland, 2018.

[6] TFL, Central London Bus Services Review: Consultation Report, Transport for London, London, UK, 2019.

[7] NYCMTA, Fast Forward: The Plan to Modernize New York City Transit, New York City Metropolitan Transit Authority, New York, NY, USA, 2019.

[8] R. F. Abenoza, O. Cats, and Y. O. Susilo, "Travel satisfaction with public transport: determinants, user classes, regional disparities and their evolution," Transportation Research Part A: Policy and Practice, vol. 95, pp. 64-84, 2017.

[9] TransitCenter, Untangling Transit: Bus Network Redesign Workshop Proceedings, TransitCenter, New York, NY, USA, 2018.

[10] A. Ceder and N. H. M. Wilson, "Bus network design," Transportation Research Part B: Methodological, vol. 20, no. 4, pp. 331-344, 1986.

[11] J. R. Current, C. S. ReVelle, and J. L. Cohon, "The hierarchical network design problem," European Journal of Operational Research, vol. 27, no. 1, pp. 57-66, 1986.

[12] Z. Y. Guo, W. Wang, and X. W. Chen, "Framework of "One road one line with straight driving public transportation system"' Journal of Transportation Systems Engineering and Information Technology, vol. 9, no. 4, pp. 159-165, 2009.

[13] H. Badia, M. Estrada, and F. Robusté, "Competitive transit network design in cities with radial street patterns," Transportation Research Part B: Methodological, vol. 59, pp. 161181, 2014.

[14] I. Dakic, L. Leclercq, and M. Menendez, "On the optimization of the bus network design: an analytical approach based on the three-dimensional macroscopic fundamental diagram," Transportation Research Part B: Methodological, vol. 149, pp. 393-417, 2021.

[15] H. Badia, J. Argote-Cabanero, and C. F. Daganzo, "How network structure can boost and shape the demand for bus transit," Transportation Research Part A: Policy and Practice, vol. 103, pp. 83-94, 2017.

[16] F. Zhao and X. Zeng, "Optimization of transit route network, vehicle headways and timetables for large-scale transit networks," European Journal of Operational Research, vol. 186, no. 2, pp. 841-855, 2008.

[17] H. Badia, M. Estrada, and F. Robusté, "Bus network structure and mobility pattern: a monocentric analytical approach on a grid street layout," Transportation Research Part B: Methodological, vol. 93, pp. 37-56, 2016.

[18] H. Chen, W. Gu, M. J. Cassidy, and C. F. Daganzo, "Optimal transit service atop ring-radial and grid street networks: a continuum approximation design method and comparisons," Transportation Research Part B: Methodological, vol. 81, no. 3, pp. 755-774, 2015.

[19] L. I. Dong-Mei and J. X. Chen, "Application of cluster analysis in evaluation of public traffic network," Journal of Hebei University of Science and Technology, pp. 279-282, 2012. 
[20] S. M. Feng and H. R. Chen, "Study of public transit network optimization method," Journal of Harbin Institute of Technology, pp. 691-693, 2005.

[21] W. Fan and R. B. Machemehl, "Optimal transit route network design problem with variable transit demand: genetic algorithm approach," Journal of Transportation Engineering, vol. 132, no. 1, pp. 40-51, 2006.

[22] F. M. Leurent and V. Benezech, "The passenger stock and attractivity threshold model for traffic assignment on a public transit network with capacity constraint," in Proceedings of the Transportation Research Board Meeting, Washington, DC, USA, May 2011.

[23] Z. Liu, "Study of network optimization method based on ACL," Procedia Engineering, vol. 15, pp. 3959-3963, 2011.

[24] S. N. Kuan, H. L. Ong, and K. M. Ng, "Solving the feeder bus network design problem by genetic algorithms and ant colony optimization," Advances in Engineering Software, vol. 37, no. 6, pp. 351-359, 2006.

[25] M. H. Baaj and H. S. Mahmassani, "An AI-based approach for transit route system planning and design," Journal of Advanced Transportation, vol. 25, no. 2, pp. 187-209, 1991.

[26] B. Yu and L. Yanbo, "Bi-level model for bus route network optimization and its solution," Journal of Jilin University (Engineering and Technology Edition), vol. 40, no. 2, pp. 402-0405, 2010.

[27] Z. Yang, B. Yu, and C. Cheng, "A parallel ant colony algorithm for bus network optimization," Computer-aided Civil \& Infrastructure Engineering, vol. 22, no. 1, pp. 44-55, 2010.

[28] S. Xiao Mei and Y. Lei, "Travel time reliability evaluation model for adjacent bus roads," Journal of Transportation Systems Engineering and Information Technology, vol. 12, no. 2, pp. 144-149, 2012.

[29] X. Zhang, E. Miller-Hooks, and K. Denny, "Assessing the role of network topology in transportation network resilience," Journal of Transport Geography, vol. 46, pp. 35-45, 2015.

[30] A. Reggiani, "Network resilience for transport security: some methodological considerations," Transport Policy, vol. 28, pp. 63-68, 2013.

[31] S. Hosseini, K. Barker, and J. E. Ramirez-Marquez, "A review of definitions and measures of system resilience," Reliability Engineering \& System Safety, vol. 145, pp. 47-61, 2016.

[32] O. Cats, G.-J. Koppenol, and M. Warnier, "Robustness assessment of link capacity reduction for complex networks: application for public transport systems," Reliability Engineering \& System Safety, vol. 167, pp. 544-553, 2017.

[33] K. Barker, J. E. Ramirez-Marquez, and C. M. Rocco, "Resilience-based network component importance measures," Reliability Engineering \& System Safety, vol. 117, pp. 89-97, 2013.

[34] X. Liang, Z. Lu, and Y. Liu, "Application of reliability theory in public transit network," Forum of Chinese Association for Science and Technology, no. 1, pp. 128-129, 2008.

[35] R. Sun, F. Lu, and W. Ming, "Analysis of Public Transit Network accessibility characteristics based on complex network," Logistics Engineering and Management, vol. 41, no. 5, pp. 150-151, 2019.

[36] F. Zhao and X. Zeng, "Optimization of transit network layout and headway with a combined genetic algorithm and simulated annealing method," Engineering Optimization, vol. 386, pp. 701-722, 2006.

[37] S. Wang, Study on the Structure and Reliability of Bus Network in Qingdao Development Zone, Shandong University of Science and Technology, Qingdao, China, 2009.
[38] M. Huang, "Topological structure analysis of multi-level bus network," Road Traffic Technology, vol. 27, no. 5, pp. 93-99, 2010.

[39] J. Zou, Study on Optimization Theory of Multi-Level Network Structure of Conventional Public Transit, Chang'an University, Xi'an, China, 2014.

[40] S. Mishra, T. F. Welch, and M. K. Jha, "Performance indicators for public transit connectivity in multi-modal transportation networks," Transportation Research Part A: Policy and Practice, vol. 46, no. 7, pp. 1066-1085, 2012.

[41] T. Aven and U. Jensen, "Stochastic models in reliability," Applications of Mathematics, vol. 41, 1999. 